\title{
Synchronization of fractional-order dynamical network via aperiodically intermittent pinning control
}

\author{
Jiaying Zhou', Jiaye $\mathrm{Yan}^{1}$ and Zhaoyan $\mathrm{Wu}^{1 *} \mathrm{~B}$
}

"Correspondence:
zhywu@jxnu.edu.cn
${ }^{1}$ College of Mathematics and
Information Science, Jiangxi Normal
University, Nanchang, P.R. China

University, Nanchang, P.R. China

\begin{abstract}
In this paper, synchronization of fractional-order network is investigated. The aperiodically intermittent pinning control scheme is adopted to design effective controllers for achieving the synchronization. Noticeably, the topology is directed and only the first node is controlled. Based on the Lyapunov function method and mathematical analysis technique, some sufficient conditions are derived and demonstrated to be effective by a numerical example.
\end{abstract}

Keywords: Synchronization; Fractional-order network; Aperiodically intermittent control; Pinning control

\section{Introduction}

Synchronization as a typical and important collective behavior of dynamical networks has been extensively investigated [1-23]. For many networks, especially those coupled with large number of nodes, they cannot synchronize themselves or synchronize with desired goals without external controls. Therefore, different control schemes, such as feedback control, intermittent control, impulsive control, pinning control, and so on, have been developed.

Intermittent control is a kind of discontinuous control consisting of work time and rest time in a sequence of intervals. In virtue of its high efficiency and strong practicability, it has been adopted to design effective controllers in practical applications, and lots of results have been obtained. For instance, the signal will become weak in transmission, so an external control should be added to increase the strength of the signal. Zhou et al. [16] studied the exponential lag synchronization for neural networks with mixed delays via intermittent control. Zhang et al. [17] considered the intermittent control for clusterdelay synchronization in directed networks. He et al. [23] investigated the exponential synchronization of dynamical network with distributed delays via intermittent control. From practical point of view, periodically intermittent control is unreasonable in some cases. As we know, the generation of wind power is typically aperiodically intermittent [8]. The heating system of central air-conditioning can run by the aperiodically intermittent operation mode (off-preheat-occupancy-off) [2]. Therefore, aperiodically intermittent control is adopted to study the synchronization of dynamical networks. Guan et al. [4] studied the

(c) The Author(s) 2019. This article is distributed under the terms of the Creative Commons Attribution 4.0 International License (http://creativecommons.org/licenses/by/4.0/), which permits unrestricted use, distribution, and reproduction in any medium, provided you give appropriate credit to the original author(s) and the source, provide a link to the Creative Commons license, and indicate if changes were made. 
cluster synchronization of coupled genetic regulatory networks with delays via aperiodically adaptive intermittent control. Liu et al. [6] studied the finite-time synchronization of delayed dynamical networks via aperiodically intermittent control. On the other hand, for large-scale networks, it is impractical to add controllers onto all nodes. Therefore, intermittent control and pinning control are combined together to design effective controllers. That is, only a fraction of network nodes is controlled. Liu and Chen [8] studied the synchronization of nonlinear coupled networks via aperiodically intermittent pinning control. Cai et al. [12] studied the outer synchronization between two hybrid-coupled delayed dynamical networks via aperiodically adaptive intermittent pinning control.

The above results mainly concentrated on integer-order dynamical networks. Compared with integer-order dynamical networks, fractional-order dynamical networks can excellently describe the memory and hereditary properties of various models. In fact, fractional-order systems, such as viscoelastic systems, dielectric polarization, electromagnetic waves, heat conduction, robotics, finance, and so on [24-30], are ubiquitous in real world. Recently, synchronization of fractional-order complex networks has been investigated as well [31-40]. Particularly, Li et al. [38] studied the synchronization of fractionalorder dynamical networks via periodically intermittent pinning control. Zhou et al. [40] studied the cluster synchronization of fractional-order directed networks via intermittent pinning control. Naturally, how to design aperiodically intermittent pinning controllers for achieving synchronization of fractional-order networks is an important issue and deserves further investigations. The main contribution of this paper is the design of aperiodically intermittent pinning controllers and the derivation of the sufficient conditions for achieving synchronization of fractional-order network. It is noted that the obtained results generalize some of the results in Refs. [8, 38]. For example, periodically intermittent pinning control of fractional-order dynamical network is generalized to aperiodically intermittent pinning control.

The paper is organized as follows. In Sect. 2, some necessary preliminaries about fractional calculus and the model of fractional-order networks are presented. In Sect. 3, aperiodically intermittent pinning controllers are designed and sufficient conditions for achieving synchronization are derived based on Lyapunov stability theory and mathematical analysis method. In Sect. 4, numerical simulation is performed to demonstrate the effectiveness of the obtained results. Finally, some conclusions are presented in Sect. 5.

Notations. For real matrix $A \in \mathbb{R}^{N \times N}, A^{s}=\left(A+A^{T}\right) / 2$ denotes the symmetric part. If all the eigenvalues of $A$ are real, let $\lambda_{\max }(A)$ and $\lambda_{\min }(A)$ be its largest and smallest eigenvalues, respectively. The symbol $\otimes$ denotes the Kronecker product.

\section{Model description and preliminaries}

In this section, some definitions, lemmas, and well-known results about fractional differential equations are recalled. In addition, the mathematic model of fractional complex network is introduced.

\subsection{Caputo fractional operator and Mittag-Leffler function}

Caputo fractional operator plays an important role in the fractional systems, since the initial conditions for fractional differential equations with Caputo derivatives take the same form as for integer-order differential, which have well-understood physical meanings [24]. Thus, we use Caputo derivatives as a main tool in this paper. The formula of the Caputo fractional derivative is defined as follows. 
Definition 1 ([24]) The Caputo fractional derivative of function $x(t)$ is defined as

$$
{ }_{0}^{C} D_{t}^{\alpha} x(t)=\frac{1}{\Gamma(m-\alpha)} \int_{0}^{t}(t-\tau)^{m-\alpha-1} x^{(m)}(\tau) d \tau,
$$

where $m-1<\alpha<m, m \in Z^{+}$. Let $m=1,0<\alpha<1$, then

$$
{ }_{0}^{C} D_{t}^{\alpha} x(t)=\frac{1}{\Gamma(1-\alpha)} \int_{0}^{t}(t-\tau)^{-\alpha} x^{\prime}(\tau) d \tau \text {. }
$$

For simplicity, denote $D_{t}^{\alpha} x(t)$ as ${ }_{0}^{C} D_{t}^{\alpha} x(t)$. The following properties of Caputo operators are specially provided.

Lemma 1 ([24]) If $w(t), u(t) \in C^{1}\left[t_{0}, b\right]$, and $\alpha>0, \beta>0$, then

(1) $D_{t}^{\alpha} D^{-\beta} w(t)=D_{t}^{\alpha-\beta} w(t)$,

(2) $D_{t}^{\alpha}(w(t) \pm u(t))=D_{t}^{\alpha} w(t) \pm D_{t}^{\alpha} u(t)$.

The Mittag-Leffler function is defined by

$$
E_{\alpha, \beta}(z):=\sum_{i=0}^{\infty} \frac{z^{i}}{\Gamma(\alpha i+\beta)}
$$

where $\alpha>0, \beta>0$, and $\Gamma(\cdot)$ is the gamma function. For short, $E_{\alpha}(z):=E_{\alpha, 1}(z)$. The following properties of Mittag-Leffler function will be used below.

Lemma $2([38,39])$ Let $V(t)$ be a continuous function on $\left[t_{0},+\infty\right)$ and satisfy

$$
D_{t}^{\alpha} V(t) \leq \theta V(t)
$$

where $0<\alpha<1, \theta$ is a constant and $t_{0}$ is the initial time, then

$$
V(t) \leq V\left(t_{0}\right) E_{\alpha}\left(\theta\left(t-t_{0}\right)^{\alpha}\right)
$$

\subsection{Model description}

Consider a fractional-order network consisting of $N$ individuals described by

$$
D_{t}^{\alpha} x_{i}(t)=f\left(x_{i}(t)\right)+b \sum_{j=1}^{N} c_{i j} \Gamma x_{j}(t), \quad i=1,2, \ldots, N
$$

where $0<\alpha<1, x_{i}(t)=\left(x_{i 1}(t), x_{i 2}(t), \ldots, x_{i n}(t)\right)^{T} \in \mathbb{R}^{n}$ is the state variable of the $i$ th node, $f$ : $\mathbb{R}^{n} \rightarrow \mathbb{R}^{n}$ is a continuously vector-valued function, $b>0$ is the coupling strength, and $\Gamma=$ $\operatorname{diag}\left(\gamma_{1}, \ldots, \gamma_{n}\right) \in \mathbb{R}^{n \times n}$ is the inner coupling matrix, $C=\left(c_{i j}\right) \in \mathbb{R}^{N \times N}$ is the zero-row-sum outer coupling matrix determining the topology and coupling strength of the network, which are defined as follows: if there exists a connection from node $j$ to node $i(i \neq j)$, then $c_{i j}>0$; otherwise, $c_{i j}=0$.

The objective here is to synchronize network (1) to the desired orbit $\eta(t)$ by designing aperiodically intermittent pinning controllers, where $\eta(t)$ is a solution of an isolated 
node satisfying $D_{t}^{\alpha} \eta(t)=f(\eta(t))$. For simplicity, only the first node is controlled and the controlled network is written as follows:

$$
\left\{\begin{aligned}
D_{t}^{\alpha} x_{1}(t)= & f\left(x_{1}(t)\right)+b \sum_{j=1}^{N} c_{1 j} \Gamma x_{j}(t) & & \\
& +b k \Gamma\left(\eta(t)-x_{1}(t)\right), & & t \in\left[t_{p}, s_{p}\right), \\
D_{t}^{\alpha} x_{i}(t)= & f\left(x_{i}(t)\right)+b \sum_{j=1}^{N} c_{i j} \Gamma x_{j}(t), & & i=2, \ldots, N, t \in\left[t_{p}, s_{p}\right), \\
D_{t}^{\alpha} x_{i}(t)= & f\left(x_{i}(t)\right)+b \sum_{j=1}^{N} c_{i j} \Gamma x_{j}(t), & & i=1, \ldots, N, t \in\left[s_{p}, t_{p+1}\right),
\end{aligned}\right.
$$

where $p=1,2,3, \ldots, k>0$ is the control gain, $0=t_{1}<s_{1}<t_{2}<s_{2}<\cdots<t_{p}<s_{p}<\cdots$. Then $s_{p}-t_{p}$ and $t_{p+1}-s_{p}$ denote the $p$ th control and rest width respectively.

Let $e_{i}(t)=x_{i}(t)-\eta(t)$ be the synchronization errors. Then the error system is

$$
\left\{\begin{aligned}
D_{t}^{\alpha} e_{1}(t)= & f\left(x_{1}(t)\right)-f(s(t))+b \sum_{j=1}^{N} c_{1 j} \Gamma e_{j}(t) & & \\
& -b k \Gamma e_{1}(t), & & t \in\left[t_{p}, s_{p}\right), \\
D_{t}^{\alpha} e_{i}(t)= & f\left(x_{i}(t)\right)-f(s(t))+b \sum_{j=1}^{N} c_{i j} \Gamma e_{j}(t), & & i=2, \ldots, N, t \in\left[t_{p}, s_{p}\right), \\
D_{t}^{\alpha} e_{i}(t)= & f\left(x_{i}(t)\right)-f(s(t))+b \sum_{j=1}^{N} c_{i j} \Gamma e_{j}(t), & & i=1, \ldots, N, t \in\left[s_{p}, t_{p+1}\right) .
\end{aligned}\right.
$$

Let $\mathscr{E}(t)=\left(e_{1}(t)^{T}, \ldots, e_{N}(t)^{T}\right)^{T}$ and

$$
F(\mathscr{E}(t))=\left(\left(f\left(x_{1}(t)\right)-f(\eta(t))\right)^{T}, \ldots,\left(f\left(x_{N}(t)\right)-f(\eta(t))\right)^{T}\right)^{T},
$$

the error system can be rewritten as

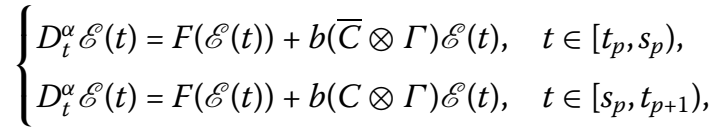

where $\bar{C}=C-\operatorname{diag}(k, 0, \ldots, 0)$.

Assumption 1 ([1]) Suppose that there exist two positive definite diagonal matrices $P=$ $\operatorname{diag}\left(p_{1}, p_{2}, \ldots, p_{n}\right)$ and $\Delta=\operatorname{diag}\left(\delta_{1}, \delta_{2}, \ldots, \delta_{n}\right)$ such that

$$
(u-v)^{T} P(f(u)-f(v)-\Delta u+\Delta v) \leq 0
$$

for any $u, v \in \mathbb{R}^{n}$.

Lemma 3 ([1]) Suppose that $C$ is an irreducible zero-row-sum matrix with nonnegative off-diagonal elements and $\mu<0$. Then there exists a positive definite diagonal matrix $\Phi=$ $\operatorname{diag}\left(\phi_{1}, \ldots, \phi_{N}\right)$ such that $C_{\mu}=C+\operatorname{diag}(\mu, 0, \ldots, 0)$ is Lyapunov stable, i.e., $\Phi C_{\mu}+C_{\mu}^{T} \Phi<0$.

Assumption 2 ([2]) For the aperiodically intermittent control strategy, there exist two positive scalars $\sigma$ and $\psi$ such that

$$
\left\{\begin{array}{l}
\inf _{p}\left(s_{p}-t_{p}\right)=\sigma>0 \\
\sup _{p}\left(t_{p+1}-s_{p}\right)=\psi<+\infty
\end{array}\right.
$$


Assumption 3 Suppose that the outer coupling matrix $C$ is irreducible and the inner coupling matrix $\Gamma$ is positive definite.

\section{Main results}

In this section, some sufficient conditions for achieving synchronization of the controlled network (2) are provided.

Theorem 1 Suppose that Assumptions 1-3 hold. The controlled network (2) with the intermittent pinning control can achieve synchronization if there exist positive constants $a_{1}$, $a_{2}$ and $0<\varpi<1$ such that the following conditions hold:

(i) $\Phi \otimes(P \Delta)+a_{1} \Phi \otimes P+b \widetilde{C}_{1} \otimes(P \Gamma) \leq 0$,

(ii) $\Phi \otimes(P \Delta)-a_{2} \Phi \otimes P+b \widetilde{C}_{2} \otimes(P \Gamma) \leq 0$,

(iii) $E_{\alpha}\left(-a_{1} \sigma^{\alpha}\right) E_{\alpha}\left(a_{2} \psi^{\alpha}\right)<\varpi$,

where $P$ and $\Delta$ are defined in (5), $\widetilde{C}_{1}=(\Phi \bar{C})^{s}, \widetilde{C}_{2}=(\Phi C)^{s}$, and $\Phi$ is defined according to Lemma 3, i.e., $\widetilde{C}_{1}<0$.

Proof Consider the following Lyapunov function:

$$
V(t)=\frac{1}{2} \mathscr{E}^{T}(t)(\Phi \otimes P) \mathscr{E}(t)
$$

Then the derivative of $V(t)$ along the trajectories of (4) satisfies the following:

When $t \in\left[t_{p}, s_{p}\right)$,

$$
\begin{aligned}
D_{t}^{\alpha} V(t) \leq & \mathscr{E}(t)^{T}(\Phi \otimes P) D_{t}^{\alpha} \mathscr{E}(t) \\
= & \mathscr{E}(t)^{T}(\Phi \otimes P)(F(\mathscr{E}(t))+b(\bar{C} \otimes \Gamma) \mathscr{E}(t)) \\
\leq & \mathscr{E}(t)^{T}[\Phi \otimes(P \Delta)] \mathscr{E}(t)+b \mathscr{E}(t)^{T}[\Phi \bar{C} \otimes(P \Gamma)] \mathscr{E}(t) \\
= & \mathscr{E}(t)^{T}\left[\Phi \otimes(P \Delta)+a_{1} \Phi \otimes P+b \widetilde{C}_{1} \otimes(P \Gamma)\right] \mathscr{E}(t) \\
& -a_{1} \mathscr{E}(t)^{T}(\Phi \otimes P) \mathscr{E}(t) \\
\leq & -a_{1} V(t) .
\end{aligned}
$$

Therefore, from Lemma 2,

$$
V(t) \leq V\left(t_{p}\right) E_{\alpha}\left(-a_{1}\left(t-t_{p}\right)^{\alpha}\right) .
$$

Similarly, when $t \in\left[s_{p}, t_{p+1}\right)$,

$$
\begin{aligned}
D_{t}^{\alpha} V(t) & \leq \mathscr{E}(t)^{T}(\Phi \otimes P) D_{t}^{\alpha} \mathscr{E}(t) \\
& =\mathscr{E}(t)^{T}(\Phi \otimes P)(F(\mathscr{E}(t))+b(C \otimes \Gamma) \mathscr{E}(t)) \\
& \leq \mathscr{E}(t)^{T}[\Phi \otimes(P \Delta)] \mathscr{E}(t)+b \mathscr{E}(t)^{T}[\Phi C \otimes(P \Gamma)] \mathscr{E}(t) \\
& =\mathscr{E}(t)^{T}\left[\Phi \otimes(P \Delta)-a_{2} \Phi \otimes P+b \widetilde{C}_{1} \otimes(P \Gamma)\right] \mathscr{E}(t)
\end{aligned}
$$




$$
\begin{aligned}
& +a_{2} \mathscr{E}(t)^{T}(\Phi \otimes P) \mathscr{E}(t) \\
\leq & a_{2} V(t)
\end{aligned}
$$

which implies

$$
V(t) \leq V\left(s_{p}\right) E_{\alpha}\left(a_{2}\left(t-s_{p}\right)^{\alpha}\right) .
$$

For $0=t_{1} \leq t<s_{1}$, from (7),

$$
V(t) \leq V(0) E_{\alpha}\left(-a_{1} t^{\alpha}\right), \quad V\left(s_{1}\right) \leq V(0) E_{\alpha}\left(-a_{1} s_{1}^{\alpha}\right) .
$$

For $s_{1} \leq t<t_{2}$, from (8) and (9),

$$
\begin{aligned}
& V(t) \leq V\left(s_{1}\right) E_{\alpha}\left(a_{2}\left(t-s_{1}\right)^{\alpha}\right) \leq V(0) E_{\alpha}\left(-a_{1} s_{1}^{\alpha}\right) E_{\alpha}\left(a_{2}\left(t-s_{1}\right)^{\alpha}\right), \\
& V\left(t_{2}\right) \leq V\left(s_{1}\right) E_{\alpha}\left(a_{2}\left(t_{2}-s_{1}\right)^{\alpha}\right) \leq V(0) E_{\alpha}\left(-a_{1} s_{1}^{\alpha}\right) E_{\alpha}\left(a_{2}\left(t_{2}-s_{1}\right)^{\alpha}\right) .
\end{aligned}
$$

For $t_{2} \leq t<s_{2}$, from (7) and (10),

$$
\begin{aligned}
V(t) & \leq V\left(t_{2}\right) E_{\alpha}\left(-a_{1}\left(t-t_{2}\right)^{\alpha}\right) \\
& \leq V(0) E_{\alpha}\left(-a_{1} s_{1}^{\alpha}\right) E_{\alpha}\left(a_{2}\left(t_{2}-s_{1}\right)^{\alpha}\right) E_{\alpha}\left(-a_{1}\left(t-t_{2}\right)^{\alpha}\right), \\
V\left(s_{2}\right) & \leq V\left(t_{2}\right) E_{\alpha}\left(-a_{1}\left(s_{2}-t_{2}\right)^{\alpha}\right) \\
& \leq V(0) E_{\alpha}\left(-a_{1} s_{1}^{\alpha}\right) E_{\alpha}\left(a_{2}\left(t_{2}-s_{1}\right)^{\alpha}\right) E_{\alpha}\left(-a_{1}\left(s_{2}-t_{2}\right)^{\alpha}\right) .
\end{aligned}
$$

For $s_{2} \leq t<t_{3}$, from (8) and (11),

$$
\begin{aligned}
V(t) \leq & V\left(s_{2}\right) E_{\alpha}\left(a_{2}\left(t-s_{2}\right)^{\alpha}\right) \\
\leq & V(0) E_{\alpha}\left(-a_{1}\left(s_{2}-t_{2}\right)^{\alpha}\right) E_{\alpha}\left(-a_{1} s_{1}^{\alpha}\right) \\
& \times E_{\alpha}\left(a_{2}\left(t_{2}-s_{1}\right)^{\alpha}\right) E_{\alpha}\left(a_{2}\left(t-s_{2}\right)^{\alpha}\right), \\
V\left(t_{3}\right) \leq & V\left(s_{2}\right) E_{\alpha}\left(a_{2}\left(t_{3}-s_{2}\right)^{\alpha}\right) \\
\leq & V(0) E_{\alpha}\left(-a_{1}\left(s_{2}-t_{2}\right)^{\alpha}\right) E_{\alpha}\left(-a_{1} s_{1}^{\alpha}\right) \\
& \times E_{\alpha}\left(a_{2}\left(t_{2}-s_{1}\right)^{\alpha}\right) E_{\alpha}\left(a_{2}\left(t_{3}-s_{2}\right)^{\alpha}\right) .
\end{aligned}
$$

By mathematical induction, for $t_{l} \leq t<s_{l}$,

$$
V(t) \leq V(0) E_{\alpha}\left(-a_{1}\left(t-t_{l}\right)^{\alpha}\right) \prod_{p=1}^{l-1} E_{\alpha}\left(-a_{1}\left(s_{p}-t_{p}\right)^{\alpha}\right) E_{\alpha}\left(a_{2}\left(t_{p+1}-s_{p}\right)^{\alpha}\right)
$$

and for $s_{l} \leq t<t_{l+1}$,

$$
\begin{aligned}
V(t) \leq & V(0) E_{\alpha}\left(a_{2}\left(t-s_{l}\right)^{\alpha}\right) E_{\alpha}\left(-a_{1}\left(s_{l}-t_{l}\right)^{\alpha}\right) \\
& \times \prod_{p=1}^{l-1} E_{\alpha}\left(-a_{1}\left(s_{p}-t_{p}\right)^{\alpha}\right) E_{\alpha}\left(a_{2}\left(t_{p+1}-s_{p}\right)^{\alpha}\right) .
\end{aligned}
$$


Assume that inequalities (13) and (14) hold when $l \leq k$. Then

$$
V\left(t_{k+1}\right) \leq V(0) \prod_{p=1}^{k} E_{\alpha}\left(-a_{1}\left(s_{p}-t_{p}\right)^{\alpha}\right) E_{\alpha}\left(a_{2}\left(t_{p+1}-s_{p}\right)^{\alpha}\right) .
$$

Now, for $t_{k+1} \leq t<s_{k+1}$,

$$
\begin{aligned}
V(t) \leq & V\left(t_{k+1}\right) E_{\alpha}\left(-a_{1}\left(t-t_{k+1}\right)^{\alpha}\right) \\
\leq & V(0) E_{\alpha}\left(-a_{1}\left(t-t_{k+1}\right)^{\alpha}\right) \\
& \times \prod_{p=1}^{k} E_{\alpha}\left(-a_{1}\left(s_{p}-t_{p}\right)^{\alpha}\right) E_{\alpha}\left(a_{2}\left(t_{p+1}-s_{p}\right)^{\alpha}\right), \\
V\left(s_{k+1}\right) \leq & V(0) E_{\alpha}\left(-a_{1}\left(s_{k+1}-t_{k+1}\right)^{\alpha}\right) \\
& \times \prod_{p=1}^{k} E_{\alpha}\left(-a_{1}\left(s_{p}-t_{p}\right)^{\alpha}\right) E_{\alpha}\left(a_{2}\left(t_{p+1}-s_{p}\right)^{\alpha}\right),
\end{aligned}
$$

and for $s_{k+1} \leq t<t_{k+2}$,

$$
\begin{aligned}
V(t) \leq & V\left(s_{k+1}\right) E_{\alpha}\left(a_{2}\left(t-s_{k+1}\right)^{\alpha}\right) \\
\leq & V(0) E_{\alpha}\left(a_{2}\left(t-s_{k+1}\right)^{\alpha}\right) E_{\alpha}\left(-a_{1}\left(s_{k+1}-t_{k+1}\right)^{\alpha}\right) \\
& \times \prod_{p=1}^{k} E_{\alpha}\left(-a_{1}\left(s_{p}-t_{p}\right)^{\alpha}\right) E_{\alpha}\left(a_{2}\left(t_{p+1}-s_{p}\right)^{\alpha}\right),
\end{aligned}
$$

that is, inequalities (13) and (14) hold for $l=k+1$. Thus, for any $l$,

$$
\begin{aligned}
V\left(t_{l}\right) & \leq V(0) \prod_{p=1}^{l-1} E_{\alpha}\left(-a_{1}\left(s_{p}-t_{p}\right)^{\alpha}\right) E_{\alpha}\left(a_{2}\left(t_{p+1}-s_{p}\right)^{\alpha}\right) \\
& \leq V(0) \prod_{p=1}^{l-1} E_{\alpha}\left(-a_{1} \sigma^{\alpha}\right) E_{\alpha}\left(a_{2} \psi^{\alpha}\right) \\
& \leq V(0) \varpi^{l-1},
\end{aligned}
$$

i.e., $V\left(t_{l}\right) \rightarrow 0$ as $l \rightarrow \infty$. And for $t_{l} \leq t<s_{l}$,

$$
V(t) \leq V\left(t_{l}\right) E_{\alpha}\left(-a_{1}\left(t-t_{l}\right)^{\alpha}\right)
$$

which implies that $V(t) \rightarrow 0$ as $t \rightarrow \infty$. Similarly, one can show that $V(t) \rightarrow 0$ as $t \rightarrow \infty$ for $s_{l} \leq t<t_{l+1}$. Then, the synchronization is achieved and the proof is completed.

Let $\kappa^{*}=\lambda_{\max }(\Phi \otimes(P \Delta)), \vartheta^{*}=\lambda_{\max }(\Phi \otimes P), \vartheta_{*}=\lambda_{\min }(\Phi \otimes P), c_{1}^{*}=\lambda_{\max }\left(\widetilde{C}_{1} \otimes(P \Gamma)\right)$, and $c_{2}^{*}=\lambda_{\max }\left(\widetilde{C}_{2} \otimes(P \Gamma)\right)$. We have the following corollary.

Corollary 1 Suppose that Assumptions 1-3 hold. The controlled network (2) with the intermittent pinning control can achieve synchronization if there exist positive constants $a_{1}$, 
$a_{2}$, and $0<\varpi<1$ such that the following conditions hold:

(i) $\kappa^{*}+a_{1} \vartheta^{*}+b c_{1}^{*} \leq 0$,

(ii) $\kappa^{*}-a_{2} \vartheta_{*}+b c_{2}^{*} \leq 0$,

(iii) $E_{\alpha}\left(-a_{1} \sigma^{\alpha}\right) E_{\alpha}\left(a_{2} \psi^{\alpha}\right)<\varpi$.

Remark 1 If we choose $t_{p+1}-t_{p}=T>0$ and $s_{p}-t_{p}=\kappa T$ with $0<\kappa<1$, then the intermittent control is periodic. And condition (iii) in Theorem 1 (or Corollary 1 ) is rewritten as $E_{\alpha}\left(-a_{1}(\kappa T)^{\alpha}\right) E_{\alpha}\left(a_{2}((1-\kappa) T)^{\alpha}\right)<\varpi<1$, which is similar to condition (iii) in Theorem 1 of Ref. [38]. That is, the obtained results generalize the results in Ref. [38] from periodic control to aperiodic control.

Remark 2 If we choose $\alpha=1$, then network (2) is an integer-order network and condition (iii) in Theorem 1 (or Corollary 1 ) is rewritten as $e^{-a_{1} \sigma} e^{a_{2} \psi}<\varpi$. Further, we have $a_{2} \psi-$ $a_{1} \sigma<\ln \varpi<0$, which is similar to the condition in Corollary 4 of Ref. [8]. That is, we generalize the results from integer-order network to fractional-order network.

\section{Numerical simulations}

Consider a fractional-order dynamical network consisting of 10 nodes. The node dynamics is chosen as the following fractional-order Chua circuit [41]:

$$
\left\{\begin{array}{l}
D_{t}^{\alpha} x_{i 1}=9\left(x_{i 2}-x_{i 1}-\varphi\left(x_{i 1}\right)\right) \\
D_{t}^{\alpha} x_{i 2}=x_{i 1}-x_{i 2}+x_{i 3} \\
D_{t}^{\alpha} x_{i 3}=-100 / 7 x_{i 2}
\end{array}\right.
$$

where $i=1,2, \ldots, 10, \alpha=0.99, \varphi\left(x_{i 1}\right)=-5 / 7 x_{i 1}-3 / 14\left(\left|x_{i 1}+1\right|-\left|x_{i 1}-1\right|\right)$.

For simplicity, choose $P$ as an identity matrix. Then one has

$$
\begin{aligned}
\left(x_{i}\right. & -s)^{T}\left(f\left(x_{i}\right)-f(s)\right) \\
& =e_{i}^{T}\left(9 e_{i 2}-9 e_{i 1}-9\left(\varphi\left(x_{i 1}\right)-\varphi\left(s_{1}\right)\right), e_{i 1}-e_{i 2}+e_{i 3},-100 / 7 e_{i 2}\right)^{T} \\
& \leq 9 / 7 e_{i 1}^{2}-e_{i 2}^{2}+10 e_{i 1} e_{i 2}-93 / 7 e_{i 2} e_{i 3} \\
& \leq\left(\frac{9}{7}+5 \rho\right) e_{i 1}^{2}+\left(-1+\frac{5}{\rho}+\frac{93 \beta}{14}\right) e_{i 2}^{2}+\frac{93}{14 \beta} e_{i 3}^{2} .
\end{aligned}
$$

Let $\rho=1.36$ and $\beta=0.83$, we can choose $\Delta=\operatorname{diag}(8.19,8.19,8.19)$ such that Assumption 1 holds. 


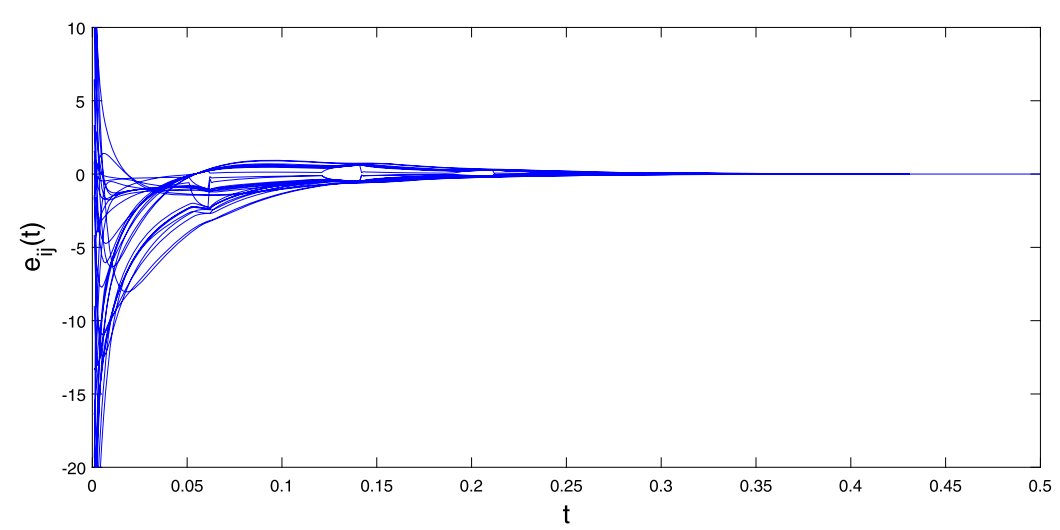

Figure 1 The orbits of the synchronization errors $e_{i j}(t), i=1,2, \ldots, 10, j=1,2,3$.

In numerical simulations, we choose $k=1, b=100, \Gamma$ as an identity matrix and

$$
C=\left[\begin{array}{cccccccccc}
-3 & 0 & 1 & 1 & 1 & 0 & 0 & 0 & 0 & 0 \\
1 & -5 & 0 & 1 & 1 & 1 & 1 & 0 & 0 & 0 \\
1 & 1 & -5 & 0 & 1 & 1 & 1 & 0 & 0 & 0 \\
1 & 1 & 0 & -4 & 1 & 0 & 1 & 0 & 0 & 0 \\
0 & 1 & 1 & 1 & -4 & 0 & 1 & 0 & 0 & 0 \\
1 & 1 & 1 & 1 & 0 & -4 & 0 & 0 & 0 & 0 \\
0 & 1 & 1 & 0 & 0 & 0 & -4 & 1 & 1 & 0 \\
1 & 0 & 0 & 0 & 0 & 0 & 0 & -2 & 0 & 1 \\
0 & 0 & 0 & 0 & 0 & 0 & 1 & 0 & -2 & 1 \\
0 & 0 & 0 & 0 & 0 & 0 & 0 & 1 & 1 & -2
\end{array}\right] .
$$

The real parts of the eigenvalues of $C$ are $0,-0.7876,-2,-3.3482,-3.615,-3.615,-4.3919$, $-4.6587,-6.2918,-6.2918$. That is, the matrix $C$ is irreducible. According to the discussions in [1], we choose

$$
\Phi=\operatorname{diag}(0.4206,0.2602,0.2409,0.2764,0.2995,0.1253,0.359,0.359,0.359,0.359)
$$

in Lemma 3. By simple calculations, we have $\kappa^{*}=3.4447, \vartheta^{*}=0.4206, \vartheta_{*}=0.1253, c_{1}^{*}=$ -0.0857 , and $c_{2}^{*}=0$. Then we choose $a_{1}=12$ and $a_{2}=27.5$ such that conditions (i) and (ii) in Corollary 1 hold. Further, we choose $\sigma=0.05, \psi=0.02$, and $\varpi=0.96$. We have $E_{\alpha}\left(-12 \sigma^{\alpha}\right) E_{\alpha}\left(27.5 \psi^{\alpha}\right)=0.9581<\varpi$, i.e., condition (iii) in Corollary 1 holds. Specially, we choose the first 7 elements of sequences $\left\{t_{p}\right\}$ and $\left\{s_{p}\right\}$ as $(0,0.06,0.14,0.21,0.3,0.36,0.42)$ and $(0.05,0.12,0.19,0.28,0.35,0.41,0.5)$. Figure 1 shows the orbits of the synchronization errors $e_{i j}(t), i=1,2, \ldots, 10, j=1,2,3$.

\section{Conclusions}

In this paper, the synchronization of complex network coupled with fractional-order dynamical systems is studied. Aperiodically intermittent control scheme is adopted to design effective controllers combining with pinning strategy. That is, only the first node is controlled. Sufficient conditions for achieving the synchronization are derived and verified by 
numerical example. Obviously, the obtained results for networks with only one controller are also valid for networks with more controllers. Therefore, in practical applications, especially for large-scale networks, more nodes are usually controlled to reduce the coupling strength and/or control gain.

\section{Acknowledgements}

The authors thank the editor and anonymous referees for their valuable suggestions and comments, which improved the presentation of this paper.

\section{Funding}

This work is jointly supported by the NSFC under Grant No. 61463022, the NSF for Distinguished Young Scholar of Jiangxi Province of China under Grant 20171BCB23031, the program of China Scholarships Council under Grant No. 201708360078, the Graduate Domestic Visiting Project of Jiangxi Normal University, and the Graduate Innovation Project of Jiangxi Normal University under Grant No. YJS2017060

\section{Availability of data and materials}

All data are fully available without restriction.

\section{Competing interests}

The authors declare that they have no competing interests.

\section{Authors' contributions}

All the authors contributed equally to this work. They all read and approved the final version of the manuscript.

\section{Publisher's Note}

Springer Nature remains neutral with regard to jurisdictional claims in published maps and institutional affiliations.

Received: 1 January 2019 Accepted: 21 April 2019 Published online: 03 May 2019

\section{References}

1. Chen, T., Liu, X.: Pinning complex networks by a single controller. IEEE Trans. Circuits Syst. I, Regul. Pap. 54, 1317-1326 (2007)

2. Liu, X., Chen, T.: Synchronization of complex networks via aperiodically intermittent pinning control. IEEE Trans. Autom. Control 60, 3316-3321 (2015)

3. Rao, P., Wu, Z., Liu, M.: Adaptive projective synchronization of dynamical networks with distributed time delays. Nonlinear Dyn. 67, 1729-1736 (2012)

4. Guan, Z., Yue, D., Hu, B.: Cluster synchronization of coupled genetic regulatory networks with delays via aperiodically adaptive intermittent control. IEEE Trans. Nanobiosci. 16, 585-599 (2017)

5. Deng, L., Wu, Z., Wu, Q.: Pinning synchronization of complex network with non-derivative and derivative coupling. Nonlinear Dyn. 73, 775-782 (2013)

6. Liu, M., Jiang, H., Hu, C.: Finite-time synchronization of delayed dynamical networks via aperiodically intermittent control. J. Franklin Inst. 354, 5374-5397 (2017)

7. Wu, Z., Duan, J., Fu, X.: Synchronization of an evolving complex hyper-network. Appl. Math. Model. 38, 2961-2968 (2014)

8. Liu, X., Chen, T.: Synchronization of nonlinear coupled networks via aperiodically intermittent pinning control. IEEE Trans. Neural Netw. Learn. Syst. 26, 113-126 (2015)

9. Liu, D., Wu, Z., Ye, Q.: Adaptive impulsive synchronization of uncertain drive-response complex-variable chaotic systems. Nonlinear Dyn. 75, 209-216 (2014)

10. Liu, X., Chen, T:: Synchronization of linearly coupled networks with delays via aperiodically intermittent pinning control. IEEE Trans. Neural Netw. Learn. Syst. 26, 2396-2407 (2015)

11. Gong, X., Wu, Z:. Adaptive pinning impulsive synchronization of dynamical networks with time-varying delay. Adv. Differ. Equ. 2015, 240 (2015)

12. Cai, S., Lei, X., Liu, Z.: Outer synchronization between two hybrid-coupled delayed dynamical networks via aperiodically adaptive intermittent pinning control. Complexity 21, 593-605 (2016)

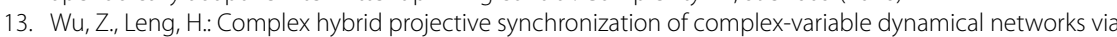
open-plus-closed-loop control. J. Franklin Inst. 354, 689-701 (2017)

14. Lei, X., Cai, S., Jiang, S., Liu, Z.: Adaptive outer synchronization between two complex delayed dynamical networks via aperiodically intermittent pinning control. Neurocomputing 222, 26-35 (2017)

15. Zhou, P., Cai, S.: Pinning synchronization of complex directed dynamical networks under decentralized adaptive strategy for aperiodically intermittent control. Nonlinear Dyn. 90, 287-299 (2017)

16. Zhou, P., Cai, S.: Adaptive exponential lag synchronization for neural networks with mixed delays via intermittent control. Adv. Differ. Equ. 2018, 40 (2018)

17. Zhang, J., Wang, Y., Ma, Z., Qiu, J., Alsaadi, F.: Intermittent control for cluster-delay synchronization in directed networks. Complexity 2018, 1069839 (2018)

18. Wu, Z., Leng, H.: Impulsive synchronization of drive-response chaotic delayed neural networks. Adv. Differ. Equ. 2016 206 (2016)

19. Xu, C., Yang, X., Lu, F., Feng, J., Alsaadi, F., Hayat, T.: Finite-time synchronization of networks via quantized intermittent pinning control. IEEE Trans. Cybern. 48, 3021-3027 (2018) 
20. Wu, X., Feng, J., Nie, Z.: Pinning complex-valued complex network via aperiodically intermittent control. Neurocomputing 305, 70-77 (2018)

21. Feng, J., Yang, P., Zhao, Y.: Cluster synchronization for nonlinearly time-varying delayed coupling complex networks with stochastic perturbation via periodically intermittent pinning control. Appl. Math. Comput. 291, 52-68 (2016)

22. Wang, J., Xu, C., Chen, M., Feng, J., Chen, G.: Stochastic feedback coupling synchronization of networked harmonic oscillators. Automatica 87, 404-411 (2018)

23. He, S., Yi, G., Wu, Z.: Exponential synchronization of dynamical network with distributed delays via intermittent control. Asian J. Control 21, 1-9 (2019)

24. Podlubny, I.: Fractional Differential Equations. Academic Press, New York (1999)

25. Butzer, P., Westphal, U.: An Introduction to Fractional Calculus. World Scientific Press, Singapore (2000)

26. Hilfer, R.: Applications of Fractional Calculus in Physics. World Scientific Press, Singapore (2000)

27. Özalp, N., Demirici, E.: A fractional order SEIR model with vertical transmission. Math. Comput. Model. 54, 1-6 (2001)

28. Laskin, N.: Fractional quantum mechanics and Lévy path integrals. Phys. Lett. A 268, 298-305 (2000)

29. Kilbas, A., Srivastava, H., Trujillo, J.: Theory and Applications of Fractional Differential Equations. Elsevier, Amsterdam (2006)

30. Ahmed, E., Elgazzar, A.: On fractional order differential equations model for nonlocal epidemics. Physica A 379 , 607-614 (2007)

31. Li, H., Hu, C., Jiang, Y., Wang, Z., Teng, Z.: Pinning adaptive and impulsive synchronization of fractional-order complex dynamical networks. Chaos Solitons Fractals 92, 142-149 (2016)

32. Li, H., Cao, J., Jiang, H., Alsaedi, A.: Finite-time synchronization of fractional-order complex networks via hybrid feedback control. Neurocomputing 320,69-75 (2018)

33. Fang, Q., Peng, J.: Synchronization of fractional-order linear complex networks with directed coupling topology. Physica A 490, 542-553 (2018)

34. Yang, S., Yu, J., Hu, C., Jiang, H.: Quasi-projective synchronization of fractional-order complex-valued recurrent neural networks. Neural Netw. 104, 104-113 (2018)

35. Wang, F., Yang, Y.: Exponential synchronization of fractional-order complex networks via pinning impulsive control. Nonlinear Dyn. 82, 1979-1987 (2015)

36. Bao, H., Park, J., Cao, J.: Synchronization of fractional-order complex-valued neural networks with time delay. Neural Netw. 81, 16-28 (2016)

37. Wang, G., Xiao, J., Wang, Y., Yi, J.: Adaptive pinning cluster synchronization of fractional-order complex dynamical networks. Appl. Math. Comput. 231, 347-356 (2014)

38. Li, H., Hu, C., Jiang, H., Teng, Z., Jiang, Y.: Synchronization of fractional-order complex dynamical networks via periodically intermittent pinning control. Chaos Solitons Fractals 103, 357-363 (2017)

39. Li, H., Jiang, Y., Wang, Z., Zhang, L., Teng, Z.: Global Mittag-Leffler stability of coupled system of fractional-order differential equations on network. Appl. Math. Comput. 270, 269-277 (2015)

40. Zhou, J., Zhao, Y., Wu, Z.: Cluster synchronization of fractional-order directed networks via intermittent pinning control. Physica A 519, 22-33 (2019)

41. Hartley, T., Lorenzo, C., Killory, H.: Chaos in a fractional order Chua's system. IEEE Trans. Circuits Syst. I, Regul. Pap. 42, 485-490 (1995)

\section{Submit your manuscript to a SpringerOpen ${ }^{\circ}$ journal and benefit from:}

- Convenient online submission

- Rigorous peer review

- Open access: articles freely available online

- High visibility within the field

- Retaining the copyright to your article

Submit your next manuscript at $\boldsymbol{s p r i n g e r o p e n . c o m ~}$ 